\title{
Planeamiento estratégico y su relación con el clima organizacional en una institución pública peruana
}

\author{
Strategic planning and its relationship with organizational climate in a \\ peruvian public institution
}

\author{
Antonio Obregón La Rosa ${ }^{1, *}$, Judith Aquino Céspedes ${ }^{1}$ \\ ${ }^{1}$ Universidad César Vallejo
}

\section{RESUMEN}

La presente investigación tuvo como objetivo general, determinar qué relación existe entre el planeamiento estratégico y el clima organizacional en el personal administrativo de una institución pública peruana, en este caso particular, en el Instituto Nacional de Innovación Agraria-INIA de Lima-Perú, durante el año 2013, donde la población estuvo constituida de 228 personas, la muestra de 143 administrativos, en los cuales se han empleado las variables: Planeamiento Estratégico y Clima Organizacional. El método empleado en la investigación fue el hipotético-deductivo. Esta investigación utilizó el diseño no experimental de nivel correlacional de corte transeccional, que recogió la información en un período específico; los instrumentos aplicados fueron: cuestionario de planeamiento estratégico, constituido por 40 preguntas con la escala de Likert y el cuestionario de clima organizacional, constituido por 40 preguntas con la escala de Likert que brindaron información acerca del planeamiento estratégico, y el clima organizacional, a través de la evaluación de sus distintas dimensiones. La investigación concluye que existe evidencia significativa para afirmar que, el planeamiento estratégico se relaciona significativamente con el clima organizacional en el personal administrativo; habiéndose determinado un coeficiente de correlación Rho de Spearman 0,988 , lo que representa un nivel de correlación alta y significativa.

Palabras clave: Planeamiento estratégico, clima organizacional, institución pública.

\begin{abstract}
The present study had the general objective, determine what relationship exists between the strategic planning and organizational climate in the administrative staff of one peruvian public institution, in this particular case, in the National Institute of Agrarian Innovation from Lima-Peru, during the year 2013, when the population was 228 people, the sample of 143 administrative, in which the variables have been used: Strategic Planning and Organizational Climate.The method used in the research was the hypothetical-deductive. This research used the non experimental correlational level transeccional court, which collected information on a specific period; the instruments applied were: Questionnaire Strategic Planning, which consisted of 40 questions on the scale of Likert and the questionnaire of organizational climate, which consisted of 40 questions in Likert scale which provided information about strategic planning, and organizational climate, through the evaluation of its different dimensions. The research concludes that there is significant evidence to affirm that, the strategic planning is significantly related to the organizational climate in the administrative staff; a Spearman Rho correlation coefficient of 0,988, having been determined representing a high and significant correlation level.
\end{abstract}

Keywords: Strategic planning, organizational climate, public institution.

Historial del artículo:

Recibido, 24 de noviembre de 2015; aceptado, 14 de junio de 2016; disponible en línea, 5 de julio de 2016

* Ingeniero en Industrias Alimentarias. Magíster en Gestión Pública de la Universidad César Vallejo.

Correo: antonnio2002@gmail.com 


\section{INTRODUCCIÓN}

Es importante indicar que la planificación estratégica es una herramienta útil y necesaria en toda organización, puesto que proporciona un marco de referencia para que se conduzca eficazmente; por ello la relevancia de ciertos aspectos, como establecer claramente cuál es la misión, las fortalezas, debilidades y amenazas; entre otros; y la que debe ser producto de la participación de su fuerza laboral (Sánchez, 2003). Ello implica que las relaciones internas se conduzcan adecuadamente, dentro de un clima organizacional adecuado, tal que aporte, sistémicamente a alcanzar los objetivos organizacionales y/o institucionales.

El personal que labora en las instituciones públicas peruanas se ve afectado por diversos factores que inciden en su eficiencia y productividad, algunos de ellos son, la falta de comunicación, la rotación del personal administrativo sin previo aviso, el choque entre los valores de la institución y los valores individuales del personal; todo ello genera un descontento que repercute en los ambientes de trabajo, desarrollando climas laborales reticentes, tensos y autoritarios que merman la eficacia y el rendimiento del personal en sus actividades, afectando entre otras cosas, la calidad de servicio que se brinda en la institución pública (Salazar, 2009).

El incipiente desarrollo de la cultura organizacional en nuestro país, origina una brecha, debido a que parte del personal no se encuentran totalmente identificados con su institución. Un verdadero desarrollo de la cultura organizacional, permitiría desarrollar climas de trabajos agradables que permitirían enlazar las necesidades de la organización con las necesidades del trabajador, por lo que el desempeño y la productividad de estos serían satisfactorios y en donde los mayores favorecidos serían los usuarios internos y externos de la institución (Alcalá, 2011).

Considerando lo señalado anteriormente, surge la necesidad de considerar la importancia del planeamiento estratégico y el clima organizacional como factores determinantes en la eficacia del personal que labora en una institución, a fin de elevar los niveles de productividad y excelencia de este último, propiciando además climas de trabajos motivantes que permitan a los trabajadores desarrollar un trabajo en equipo para lograr los objetivos de la organización.

En función de lo expuesto, surgen interrogantes que son propicios de ser investigados y diagnosticados para proponer estrategias y alternativas que mejoren ambas variables.

En tal sentido, la presente investigación tiene como objetivos: analizar, investigar, diagnosticar y determinar qué relación existe entre el planeamiento estratégico (diseño, implementación, monitoreo, evaluación) y el clima organizacional, en el personal administrativo de una institución pública peruana, en este caso particular, la investigación se realizó en el Instituto Nacional de Innovación Agraria-INIA de Lima-Perú.

\section{MATERIAL Y MÉTODOS}

La hipótesis fue: El planeamiento estratégico, con sus cuatro dimensiones (diseño, implementación, monitoreo y evaluación) se relaciona significativamente con el clima organizacional en el personal administrativo del Instituto Nacional de Innovación Agraria.

Las variables a estudiar fueron el planeamiento estratégico en sus cuatro dimensiones: diseño, implementación, monitoreo y evaluación; y el clima organizacional.

En el análisis de datos se aplicó la estadística descriptiva, para la contrastación de las hipótesis se aplicó la prueba no paramétrica Rho de Spearman (Álvarez, 1994).

El tipo de estudio fue básica, con un enfoque cuantitativo correlacional, con un diseño no experimental.

La población estuvo constituida por 228 trabajadores del Instituto Nacional de Innovación Agraria, de los cuales 113 fueron personal nombrado y 115 personal contratado. Para la extracción de la muestra se aplicó un Muestro Aleatorio Simple (MAS), de forma estratificada (Álvarez, 1994). Los criterios de inclusión fueron: ser personal en cada uno de los regímenes en la institución; haber asistido el día de la encuesta. Entre los criterios de exclusión, se consideró, no haber asistido el día de la encuesta.

Respecto al método empleado, la presente investigación utilizó el hipotético deductivo, el cual permitió contrastar las hipótesis a través de un diseño estructurado (Hernández, 2006).

Para la recolección de datos se utilizó la técnica de encuestas, mediante dos cuestionarios que fueron proporcionados a cada trabajador, uno para planeamiento estratégico y el otro para medir el clima organizacional, los cuales fueron validados mediante el juicio de expertos y sometidos a confiabilidad mediante el alfa de crombach (Alcalá, 2011). Los cuestionarios estuvieron compuestos por 40 preguntas, en la escala de Likert (Acero, 2013).

El cuestionario de planeamiento estratégico, tuvo el objetivo de evaluar la percepción de la calidad del planeamiento estratégico, aplicado a los trabajadores administrativos. 
El cuestionario sobre clima organizacional, tuvo el objetivo de identificar un parámetro a nivel general sobre la percepción que los individuos tienen dentro de la organización y la organización sobre ellos. Adicionalmente proporcionar retroinformación acerca de los procesos que determinan los comportamientos organizacionales, permitiendo introducir cambios planificados tanto en las actitudes y conductas de los miembros, como en la estructura organizacional o en uno o más de los subsistemas que la componen.

La validez de los instrumentos estuvo dada por el

Tabla 1: Resultados de confiabilidad del cuestionario de planeamiento estratégico.

\begin{tabular}{lcc}
\hline $\begin{array}{c}\text { Dimensión de } \\
\text { Evaluación }\end{array}$ & $\begin{array}{c}\text { Alfa de } \\
\text { Crombach }\end{array}$ & $\begin{array}{c}\mathrm{N}^{\circ} \text { de } \\
\text { Elementos }\end{array}$ \\
\hline Diseño & 0,841 & 10 \\
Implementación & 0,863 & 10 \\
Monitoreo & 0,855 & 10 \\
Evaluación & 0,951 & 10 \\
\hline
\end{tabular}

Tabla 2: Resultados de confiabilidad del cuestionario de clima organizacional.

\begin{tabular}{lcc}
\hline $\begin{array}{l}\text { Dimensión de } \\
\text { Evaluación }\end{array}$ & $\begin{array}{c}\text { Alfa de } \\
\text { Crombach }\end{array}$ & $\begin{array}{c}\mathrm{N}^{\circ} \text { de } \\
\text { Elementos }\end{array}$ \\
\hline $\begin{array}{l}\text { Relaciones } \\
\text { interpersonales }\end{array}$ & 0,921 & 5 \\
$\begin{array}{l}\text { Estilo de } \\
\text { dirección }\end{array}$ & 0,747 & 5 \\
$\begin{array}{l}\text { Sentido de } \\
\text { pertenencia }\end{array}$ & 0,758 & 5 \\
$\begin{array}{l}\text { Retribución } \\
\begin{array}{l}\text { Disponibilidad } \\
\text { de recursos }\end{array}\end{array}$ & 0,750 & 5 \\
$\begin{array}{l}\text { Estabilidad } \\
\begin{array}{l}\text { Claridad y } \\
\text { coherencia en }\end{array}\end{array}$ & 0,722 & 5 \\
la dirección & 0,849 & 5 \\
$\begin{array}{l}\text { Valores } \\
\text { Colectivos }\end{array}$ & 0,727 & 5 \\
\hline
\end{tabular}

juicio de expertos y fue corroborado con la validación de los instrumentos. El instrumento se sometió a la confiabilidad mediante el alfa de crombach (Corral, 2009).

Se observa, que los valores del Coeficiente Alfa de Crombach de los resutados de confiabilidad del cuestionario de planeamiento estratégico y clima organizacional (tablas 1,2) tienen valores superiores a 0,80 lo que indicaría que el grado de confiabilidad del instrumento es buena (Corral, 2009).
Para el análisis de datos se utilizó el programa SPSS V. 22, porcentajes en tablas y figuras para presentar la distribución de los datos, la estadística descriptiva, para la ubicación dentro de la escala de medición, para la contrastación de las hipótesis se aplicó la estadística no paramétrica mediante el coeficiente de Pearson y Rho Spearman (Álvarez, 1994).

\section{RESULTADOS}

Tabla 3: Resultados promedio de evaluación del cuestionario de planeamiento estratégico.

\begin{tabular}{lc}
\hline $\begin{array}{c}\text { Dimensión de } \\
\text { Evaluación }\end{array}$ & $\begin{array}{c}\text { Resultado promedio } \\
\text { de la encuesta }\end{array}$ \\
\hline Diseño & 2,94 \\
Implementación & 3,26 \\
Monitoreo & 2,95 \\
Evaluación & 3,41 \\
\hline
\end{tabular}

Tabla 4: Resultados promedio de evaluación del cuestionario de planeamiento estratégico.

\begin{tabular}{lc}
\hline $\begin{array}{c}\text { Dimensión de } \\
\text { Evaluación }\end{array}$ & $\begin{array}{c}\text { Resultado promedio } \\
\text { de la encuesta }\end{array}$ \\
\hline Relaciones interpersonales & 3,30 \\
Estilo de dirección & 3,37 \\
Sentido de pertenencia & 3,43 \\
Retribución & 3,27 \\
Disponibilidad de recursos & 3,28 \\
Estabilidad & 3,03 \\
$\begin{array}{l}\text { Claridad y coherencia en la } \\
\text { dirección }\end{array}$ & 3,26 \\
Valores colectivos & 3,36 \\
\hline
\end{tabular}

En las tablas 3 y 4 se presentan los resultados promedios de evaluación de los cuestionarios de planeamiento estratégico y clima organizacional, donde se observa que ambas variables se encuentran entre los valores medio de acuerdo a la Escala de Likert.

Es preciso señalar, qué de acuerdo a los resultados, las estrategias y recursos de la institución deben priorizarse en el diseño y monitoreo del Plan estratégico.

En la tabla 4 se presentan los resultados promedios de evaluación del cuestionario de clima organizacional, donde se observa que los valores promedios se encuentran entre los valores medio de acuerdo a la Escala de Likert.

Con relación al planeamiento estratégico, las 
Tabla 5: Resultados de la relación entre el planeamiento estratégico y clima organizacional en el personal administrativo del Instituto Nacional de Innovación Agraria. Lima, 2013.

\begin{tabular}{|c|c|c|c|c|c|c|c|c|}
\hline \multirow{3}{*}{$\begin{array}{c}\text { Planeamiento } \\
\text { Estratégico }\end{array}$} & \multicolumn{8}{|c|}{ Clima Organizacional } \\
\hline & \multicolumn{2}{|c|}{ Deteriorado } & \multicolumn{2}{|c|}{ Regular } & \multicolumn{2}{|c|}{ Bueno } & \multicolumn{2}{|c|}{ Total } \\
\hline & $\mathrm{n}$ & $\%$ & $n$ & $\%$ & $\mathrm{n}$ & $\%$ & $n$ & $\%$ \\
\hline Ineficaz & 40 & 28,0 & 2 & 1,4 & 0 & 0,0 & 42 & 29,4 \\
\hline Regular & 0 & 0,0 & 61 & 42,7 & 0 & 0,0 & 61 & 42,7 \\
\hline Eficaz & 0 & 0,0 & 0 & 0,0 & 40 & 28,0 & 40 & 28,0 \\
\hline Total & 40 & 28,0 & 63 & 44,1 & 40 & 28,0 & 143 & 100,0 \\
\hline
\end{tabular}

estrategias y recursos de la institución deben priorizarse en el diseño y monitoreo del Plan estratégico.

Respecto al clima organizacional, las estrategias y recursos para su mejora debe priorizarse siendo en primer lugar la estabilidad, luego la claridad y coherencia en la dirección y en la retribución.

En la figura 1 y tabla 5; se presentan los resultados

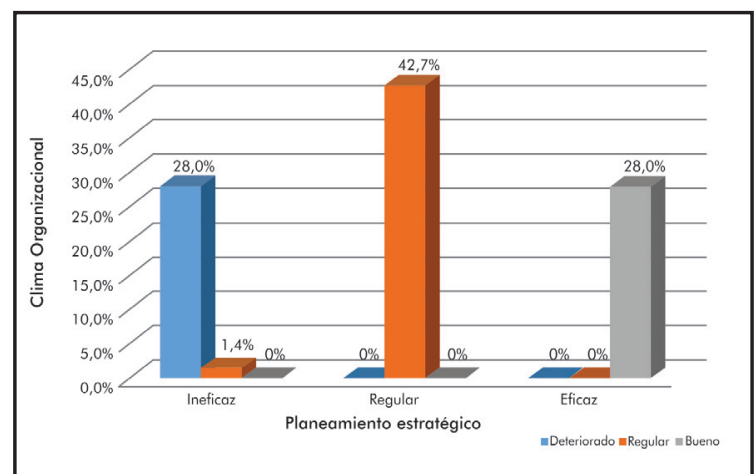

Figura 1: Relación entre Planeamiento estratégico y clima organizacional.

de la relación entre planeamiento estratégico y clima organizacional. Se observa que el planeamiento estratégico a un nivel de ineficaz, el $28,0 \%$ de los empleados encuentra un clima organizacional de nivel deteriorado, asimismo, en un nivel regular, el $42,7 \%$ de los empleados encuentra un clima organizacional de nivel regular. De otro lado, en un nivel de planeamiento estratégico eficaz, el 28,0 \% de los empleados encuentra un clima organizacional de nivel bueno.

Es preciso señalar, que esta misma relación directa se encontró para las comparaciones entre las dimensiones del planeamiento estratégico (diseño, implementación, monitoreo, evaluación) con el clima organizacional.

En la tabla 6, se presenta los resultados obtenidos de la medición de la correlación entre la variable planeamiento estratégico y clima organizacional. El resultado de 0,988 indica que existe relación positiva entre las variables, además, se encuentra en el nivel de correlación alta (Álvarez, 1994), por lo cual, se rechaza la hipótesis nula y se acepta la hipótesis general; que considera que el planeamiento estratégico se relaciona significativamente con el clima organizacional en el personal administrativo de una institución pública peruana.

\section{DISCUSIÓN}

La presente investigación refuerza lo planteado por Mendoza (2012), puesto que al haberse demostrado la correlación entre el planeamiento estratégico y el clima organizacional (Rho Spearman $=0,988$ ); se corrobora que el clima organizacional no solamente se relaciona con el desarrollo de la gestión de las instituciones; sino con la forma como estas se gestionan a través de diversas técnicas, como lo es, el planeamiento estratégico.

Asimismo, la presente investigación corrobora lo planteado por Ávila (2012), puesto que el planeamiento estratégico, partiendo de ser

Tabla 6: Correlación entre la variable planeamiento estratégico y clima organizacional.

\begin{tabular}{lllcc}
\hline & & $\begin{array}{c}\text { Clima } \\
\text { Organizacional }\end{array}$ & $\begin{array}{c}\text { Planeamiento } \\
\text { Estratégico }\end{array}$ \\
\hline \multirow{6}{*}{ Rho de } & Clima & Coeficiente de correlación & 1,000 & $0,988^{* *}$ \\
Spearman & Sig. (bilateral) & $-\cdots-----$ & 0,000 \\
& & $N$ & 143 & 143 \\
& Planeamiento & Coeficiente de correlación & $0,988^{* *}$ & 1,000 \\
& Estratégico & Sig. (bilateral) & 0,000 & $-\cdots----$ \\
& $N$ & 143 & 143 \\
\hline
\end{tabular}

**La correlación es significativa al nivel 0,01 (bilateral). 
herramienta, en la que toda organización, debe haber aportado para su elaboración; se relaciona con una serie de variables en el campo empresarial, como lo es el clima organizacional; habiendo demostrado estadísticamente un coeficiente de correlación alto y significativo (Rho Spearman=0,988); ello implica, como lo refiere Ávila (2012), que para llevar a cabo el Plan estratégico se realiza un análisis externo bajo los lineamientos políticos, económicos, sociales, tecnológicos y ecológicos que permitan tener una visión clara del entorno sobre el cual se desarrolla el sector en estudio.

La presente investigación refuerza lo planteado por Tarazona (2009), puesto que el planeamiento estratégico, debiendo ser consecuencia de la concertación y participación de la fuerza laboral; necesariamente tiene que tener algún tipo de relación con el clima organizacional que se vive en la organización; siendo que si existe dicha relación, como ha quedado demostrado en la presente investigación.

De otro lado, la presente investigación corrobora lo planteado por Zúñiga (2010), puesto que el clima organizacional se correlaciona con una serie de variables en el proceso de gestión, entre ellos el planeamiento estratégico; toda vez que se obtuvo una correlación Rho de Spearman alta y significativa $(r=0,988)$, siendo que la eficacia de su propuesta dependerá del liderazgo de los directivos de la institución.

En conclusión, la presente investigación demuestra que el planeamiento estratégico se relaciona significativamente con el clima organizacional en el personal administrativo del Instituto Nacional de Innovación Agraria; habiéndose calculado un coeficiente de correlación Rho de Spearman de 0,988 , lo que representa un nivel de correlación alta y significativa, siendo el $p<0,005$ se rechaza la hipótesis nula y se acepta la hipótesis específica.

\section{REFERENCIAS BIBLIOGRÁFICAS}

Acero , Y., Echeverri , L., Lizarazo , S., Quevedo, A., \& Sanabria, B. (mayo de 2013). Escala de Clima Organizacional (EDCO). Santafé de Bogotá, D.C. Fundación Universitaria Konrad Lorenz. Obtenido de http://www.monografias.com/trabajos35/ escalaclima

Alcalá , M. (2011). El Clima Organizacional en una Institución Pública de Educación Superior [Tesis]. México: . Campus. Tesis, Universidad del Papaloapan, Tuxtepec, Papaloapan.

Álvarez, R. (1994). Estadística Multivalente y No Paramétrica con SPSS. Madrid: Díaz de Santos.

Ávila, B. (2012). Planeamiento estratégico del sector bancario peruano. Tesis, Pontificia Universidad Católica del Perú, Lima.

Corral , Y. (2009). Validez y confiabilidad de los instrumentos de investigación para la recolección de datos. Revista ciencias de la educación, 19(33), 228-247.

Hernández , R., Fernández, C., \& Baptista , L. (2006). Metodología de la investigación (Vol. (5a Ed.)). México: Mc. Graw-Hill. Interamericana.

Mendoza, A. (2012). Clima institucional y organizacional en una institución educativa. Tesis, Universidad Cesar Vallejo, Lima.

Salazar , J., Guerrero, J., Machado, Y., \& Cañedo, R. (2009). Clima y cultura organizacional: dos componentes esenciales en la productividad laboral . Acimed, 20(4), 67-75.

Sánchez, A. (2003). Constitución política, acuerdo nacional y planeamiento estratégico en el Perú. Santiago de Chile: United Nations Publications Vol. 30.

Tarazona, O. (2009). El planeamiento estratégico y el nivel de satisfacción laboral de los docentes en las instituciones educativas del distrito de Puente Piedra. Tesis, Universidad Nacional Mayor de San Marcos, Lima.

Zúñiga, M. (2010). Estilo de liderazgo de los decanos y clima organizacional en la Universidad Nacional del Callao. Tesis, Universidad Nacional del Callao, Lima. 